\title{
PLAYFUL BOOLEAN ALGEBRAS
}

\author{
BOBAN VELIČKOVIĆ
}

\begin{abstract}
We show that for an atomless complete Boolean algebra $B$ of density $\leq 2^{\aleph_{0}}$, the Banach-Mazur, the split and choose, and the Ulam game on $B$ are equivalent. Moreover, one of the players has a winning strategy just in trivial cases: Empty wins iff $B$ adds a real; Nonempty wins iff $B$ has a $\sigma$-closed dense set. This extends some previous results of Foreman, Jech, and Vojtás.
\end{abstract}

0. Introduction. In [Je1 and Je3] Jech initiated the study of game-theoretic properties of Boolean algebras. There are mainly two sources of ideas for defining these games. One is to consider classical games of Banach, Mazur, Mycielski, Ulam and others and to translate them into the Boolean algebraic context. For the origin of these games see the Scottish book [Ma, problems 43 and 67]. The other is to look at some well-known properties of Boolean algebras, such as various distributivity conditions, the existence of dense sets with certain closedness properties, Axiom A, properness and others (these can usually be characterized by properties of the corresponding forcing extensions) and to try to devise games that would reflect them. We are mainly interested in characterizing those Boolean algebras in which one of the players has a winning strategy in a certain game. The hope is that by doing this some new and interesting concepts and problems would emerge that would improve our understanding of the structure of Boolean algebras.

Most of the results of this paper were motivated by a list of problems from [Je3]. Some of them we solve, and to others we give partial solutions. For example, we prove that if the Nonempty player has a winning strategy in the cut \& choose game on a complete Boolean algebra $B$ which has a dense set of size $\leq 2^{\aleph_{0}}$, then $B$ has a $\sigma$-closed dense set. By this we improve a previous result of Foreman [Fo], and Vojtás [Vo2], though their ideas are essential ingredients of our proof. We also give a consistent example of a poset $P$ such that $P \times P$ is equivalent to $C_{\omega_{1}}$ (the usual Cohen poset for adding a subset of $\omega_{1}$ ) but $P$ is not. By a result from [Fo] this cannot happen under $\mathrm{CH}$.

As an application we give a proof of the following result of Gregory announced in $[\mathbf{G r}]$. If "ZFC $+\exists$ weakly compact cardinal" is consistent, then so is "ZFC+GCH+ Every $\aleph_{2}$-Suslin tree is essentially $\sigma$-closed".

We show that Nonempty may or may not have a winning strategy in the both players cut \& choose game on Prikry forcing, depending on the model of set theory. Since, as is easily seen, Nonempty wins the ordinary cut \& choose game on Prikry forcing; this shows that it sometimes makes a difference if we require Nonempty to cut, too.

Received by the editors May 15, 1985.

1980 Mathematics Subject Classification. Primary 03G05, 06E05, 06E10; Secondary $03 E 55$.

Key words and phrases. Boolean algebras, infinite games, trees, measurable cardinals. 
Our terminology is mostly standard or self-explanatory. Our main interest is in complete Boolean algebras, since this seems to be the most natural setting. However, most games we consider can be defined on arbitrary Boolean algebras, indeed even partially ordered sets. We shall sometimes state the more general hypothesis under which the result is true. Instead of constructing a complete Boolean algebra we usually construct a partially ordered set, and the algebra is the (unique) completion of it. We say that two posets are equivalent (denoted by $\simeq$ ) if they have isomorphic completions. law:

Recall that a Boolean algebra $B$ is $(\kappa, \lambda)$-distributive iff it satisfies the following

$$
\bigwedge_{\alpha<\kappa \beta<\lambda} \bigvee_{\beta} a_{\alpha \beta}=\bigvee_{f: k \rightarrow \lambda} \bigwedge_{\alpha<\kappa} a_{\alpha, f(\alpha)} .
$$

An algebra is $\kappa$-Baire iff it is $(\kappa, \infty)$-distributive. A poset is $\kappa$-Baire iff its completion is.

We leave the formal definition of a (winning) strategy to the reader's imagination and satisfy ourselves with informal descriptions. If $B$ is a Boolean algebra let $B^{+}$ denote $B \backslash\{0\}$. For $b \in B^{+}, B \backslash b$ is the algebra restricted to $b$. A tree is a partially ordered set such that the predecessors of every point are well-ordered. We say that a tree is normal if every element has arbitrary high elements above it and elements on limit levels are uniquely determined by their sets of predecessors. It is usually no loss of generality to assume, as we shall, that the tree in question is normal. If $T$ is a tree and $X \subseteq$ height $(T), T \uparrow X$ is the restriction of $T$ to $X$; i.e., $T \uparrow X=\{t \in T \mid$ level $(t) \in X\}$. Let us warn the reader that some of our trees grow downwards in order to be consistent with our other notation, but this will hopefully cause no confusion.

1. The Banach-Mazur game. Consider the following version of the wellknown Banach-Mazur game played on a partially ordered set.

Players Empty and Nonempty alternately play a descending sequence of elements of a poset $P$ :

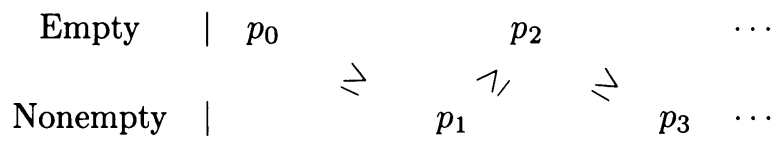

Nonempty wins the run of the game iff $\exists p \in P \forall n<\omega p \leq p_{n}$. We call this game $\mathcal{G}(P)$. It is easy to see that if $P$ is a dense subset of $\mathcal{Q}$, then $\mathcal{G}(P)$ and $\mathcal{G}(\mathcal{Q})$ are equivalent. So we may restrict ourselves to complete Boolean algebras. Jech [Je1] showed that Empty has a winning strategy in $\mathcal{G}(B)$ if $B$ is not $\left(\aleph_{0}, \infty\right)$-distributive. Note that if $B$ has a $\sigma$-closed dense set, then Nonempty wins easily-he simply plays elements of that dense set. One of the problems from [Je3] asks if the converse is true. In this section we slightly improve some partial results of Foreman [Fo] and Vojtás [Vo2].

THEOREM 1.1. Let $T$ be a tree. If Nonempty has a winning strategy in $\mathcal{G}(T)$, then $T$ has a $\sigma$-closed dense set.

PROOF. We may without loss of generality assume that $T$ is normal. Let the height (or depth) of $T$ be $\kappa$. For $\xi<\kappa, T_{\xi}$ denotes the $\xi$ th level of $T$. We first 
prove the theorem, assuming that $T$ is $<\kappa$-Baire. Later we show that the general case can be reduced to this.

Let us fix a winning strategy $\sigma$ for Nonempty in $\mathcal{G}(T)$. For $t \in T$ and $\vec{s} \subseteq T$ a finite sequence of even length we say that $\vec{s}$ is a partial play towards $t$ if $\vec{s}=\left\langle a_{i}\right| i<$ $2 k\rangle$ is played according to $\sigma$ and $a_{0} \geq a_{1} \geq \cdots \geq a_{2 k-1} \geq t$. Call $t \in T$ good if for every partial play $\vec{s}$ towards $t$ and $t^{\prime} \in T$ such that inf $\vec{s} \geq t^{\prime}>t$ there is a partial play $\vec{s}^{\prime}$ towards $t$ extending $\vec{s}$ with $\inf \vec{s}^{\prime} \leq t^{\prime}$. It can be shown (see [Fo, p. 720]) that the set of good $t$ 's is $\sigma$-closed. So we shall be done if we show that it is dense.

Now, call $\left(t, t^{\prime}\right) \in T^{2}$ a good pair if $t^{\prime}>t$ and for every partial play $\vec{s}$ towards $t^{\prime}$ there is a partial play towards $t, \vec{s}^{\prime}$ extending $\vec{s}$ such that inf $\vec{s}^{\prime} \leq t^{\prime}$.

Claim. For every $p \in T$ there is $t<p$ such that $(t, p)$ is a good pair.

Assuming the claim for a moment, let us see why this finishes the proof. For $p \in T$, build a sequence $\left\langle t_{i} \mid i<\omega\right\rangle$ such that $t_{0}=p$ and $\forall i<\omega\left(t_{i+1}, t_{i}\right)$ is a good pair. Then, by normality of $T, \inf \left\{t_{i} \mid i<\omega\right\}$ exists and is easily seen to be good.

To prove the claim, fix $p \in T$. For $\vec{s}$ a partial play towards $p$ consider the set

$$
D_{\vec{s}}=\left\{q \in T \mid \exists \vec{s}^{\prime} \text { partial play extending } \vec{s} \& q \leq \inf \vec{s}^{\prime}\right\} .
$$

Clearly, each $D_{\vec{s}}$ is dense and open below $p$. There are only $[\mathrm{ht}(p)]^{<\omega}$ partial plays towards $p$. Since $T$ is $<\kappa$-Baire, the set

$$
D_{p}=\bigcap\left\{D_{\vec{s}} \mid \vec{s} \text { is a partial play towards } p\right\}
$$

is dense below $p$. Clearly, any $t \in D_{p}$ works. The claim is proved.

Consider now the general situation-i.e., $T$ need not be $<\kappa$-Baire. By passing to a maximal antichain, we may assume without loss of generality that there is some $\lambda \leq \kappa$ such that $T$ is $<\lambda$-Baire and there is a decreasing sequence of $\lambda$ dense open sets $\left\{D_{\xi} \mid \xi<\lambda\right\}$ in $T$ such that $\bigcap\left\{D_{\xi} \mid \xi<\lambda\right\}=\varnothing$. For each $\xi<\lambda$ pick by transfinite recursion a maximal antichain $A_{\xi} \subseteq D_{\xi}$ so that if $\eta<\xi$ then $A_{\xi}$ refines $A_{\eta}$; i.e., $\forall t \in A_{\xi} \exists s \in A_{\eta}(t \leq s)$. Now, look at $R=\bigcup\left\{A_{\xi} \mid \xi<\lambda\right\}$. We claim that $R$ is dense in $T$. If $t \in T$ there is some $\xi<\lambda$ such that $t \notin D_{\xi}$. If $s$ is any element of $A_{\xi}$ compatible with $t$, then we must have $s \leq t$.

$R$ with the induced ordering is a tree of height (or depth!) $\lambda$ such that $R$ is $<\lambda$-Baire. So, by the above special case of our theorem $R$ has a $\sigma$-closed dense set. This set is dense in $T$, too.

The following lemma was also proved by Vojtás [Vo2]; we include it here for completeness.

LEMMA 1.2. Let $B$ be a complete Boolean algebra which has a dense set of size $\leq 2^{\aleph_{0}}$. Assume that Nonempty wins $\mathcal{G}(B)$. Then $B$ has a dense subset which is a tree under the induced ordering.

Proof. Let $P$ be a fixed dense subset of $B$ of size $\leq 2^{\aleph_{0}}$. Let $\kappa$ be the least cardinal such that $B$ is not $\kappa$-Baire. As in the previous proof we may assume without loss of generality that there are maximal antichains in $\mathcal{P}, A_{\xi}$, for $\xi<\kappa$, such that $A_{\xi}$ refines $A_{\eta}$ for $\eta<\xi$ and $\bigwedge_{\xi<\kappa} f(\xi)=0$ for every $f \in \prod_{\xi<\kappa} A_{\xi}$.

Claim. $\forall p \in P \exists \xi<\kappa$ such that $A_{\xi}(p)=\left\{q \in A_{\xi} \mid p \wedge q \neq 0\right\}$ has size $2^{\aleph_{0}}$.

PROOF. Let $\sigma$ be a winning strategy for Nonempty in $\mathcal{G}(P)$. Construct by induction two Cantor trees of elements of $P,\left\langle p_{s} \mid s \in 2^{<\omega}\right\rangle$ and $\left\langle q_{s} \mid s \in 2^{<\omega}\right\rangle$. Set 
$q_{\langle\rangle}=p$. If we have constructed $p_{s}$, let $q_{s-0}$ and $q_{s-1}$ be incompatible extensions of $p_{s}$ which are below different elements of $A_{\xi}$, for some $\xi_{s}<\kappa$. Then set

$$
p_{s^{-} \varepsilon}=S\left(q_{\langle\rangle}, p_{\langle\rangle}, q_{\langle s(0)\rangle} p_{\langle s(0)\rangle}, \ldots, p_{s}, q_{s^{-\varepsilon}}\right), \quad \text { for } \varepsilon=0,1 \text {. }
$$

Since $\sigma$ is a winning strategy for Nonempty, for each $f \in 2^{\omega}$ there is $p_{f}$ such that $\forall n<\omega p_{f} \leq p_{f \mid n}$. If we now let $\xi=\sup \left\{\xi_{s} \mid s \in 2^{<\omega}\right\}$, since $A_{\xi}$ 's refine each other, it is clear that $A_{\xi}(p)$ has size $2^{\aleph_{0}}$. The claim is proved.

Now, for each $\xi<\kappa$ let $E_{\xi}=\left\{p \in P|| A_{\xi}(p) \mid=2^{\aleph_{0}}\right\}$. By a simple induction argument there is a 1-1 function $\varphi_{\xi}: E_{\xi} \rightarrow A_{\xi}$ such that $\forall p \in E_{\xi} p \wedge \varphi_{\xi}(p) \neq 0$. Choose for each $p \in E_{\xi}, q_{p} \leq p \wedge \varphi_{\xi}(p)$. Extend $\left\{q_{p}: p \in E_{\xi}\right\}$ to a maximal antichain $C_{\xi}$ refining $A_{\xi}$. Next, build recursively maximal antichains $T_{\xi}$ for $\xi<\kappa$ such that $T_{\xi+1}$ refines $C_{\xi}$ and $T_{\eta}$ for $\eta \leq \xi$. Also, make sure that for limit $\xi$

$$
T_{\xi}=\left\{\bigwedge_{\eta<\xi} f(\eta) \mid f \in \prod_{\eta<\xi} T_{\eta} \text { and } \bigwedge_{\eta<\xi} f(\eta) \neq 0\right\} .
$$

Note that we do not require $T_{\xi}$ 's to be subsets of $P$. Finally, let $T=\bigcup\left\{T_{\xi} \mid \xi<\kappa\right\}$. It follows from our construction that $T$ is the required tree.

COROLlaRY 1.3. Let $B$ be a complete Boolean algebra which has a dense subset of cardinality $\leq 2^{\aleph_{0}}$. Assume that Nonempty wins $\mathcal{G}(B)$. Then $B$ has a $\sigma$-closed dense set.

This corollary was proved by Foreman [Fo] under $\mathrm{CH}$ and by Vojtás [Vo2] under a slight additional assumption. For $\kappa$ a regular cardinal let $C_{\kappa}$ be the usual complete Boolean algebra for adding a Cohen subset of $\kappa$. Similarly to [Fo] we have the following result which was also proved by Vojtáš [Vo2].

COROLlaRY 1.4. Let $B$ be a complete subalgebra of $C_{\omega_{1}}$. If $B$ is nowhere $\omega_{1}$ Baire, then $B \simeq C_{\omega_{1}}$.

Proof. By [Fo], if Nonempty wins $\mathcal{G}(C)$ and $B$ is a complete subalgebra of $C$, then Nonempty also wins $\mathcal{G}(B)$. The rest follows from Theorem 1.1 and Lemma 1.2 , since the tree constructed in the proof of Lemma 1.2 will have height $\omega_{1}$. It is a well-known fact that if $T$ is a $\sigma$-closed nonatomic tree of height $\omega_{1}$ and size $2^{\aleph_{0}}$, then the completion of $T$ is isomorphic to $C_{\omega_{1}}$.

The restriction that $B$ is nowhere $\omega_{1}$-Baire cannot be omitted, since if $2^{\aleph_{0}}=2^{\aleph_{1}}$, then $C_{\omega_{1}} \times C_{\omega_{2}} \simeq C_{\omega_{1}}$.

We now give a consistent example of a poset $P$ such that $P \times P \simeq C_{\omega_{1}}$, but $P \not C_{\omega_{1}}$. For that we have to assume $\square_{\omega_{1}}+\diamond\left\{\alpha<\omega_{2} \mid \operatorname{cof}(\alpha)=\omega_{1}\right\}+\neg \mathrm{CH}$. This is easily shown to be consistent with ZFC. Namely, using the fact that $\square_{\omega_{1}}$ and $\diamond\left\{\alpha<\omega_{2} \mid \operatorname{cof}(\alpha)=\omega_{1}\right\}$ hold in the constructible universe $L$ (see [De]), one simply adds $\aleph_{2}$ Cohen reals to a model of $V=L$. In fact we need only a weaker form of $\square$, but we shall not even bother to state it.

Let us mention that this example is related to an (equivalent) version of Jech's problem: assuming $P \times \mathcal{Q}$ has a $\sigma$-closed dense set, does $P$ have a $\sigma$-closed dense set. For the equivalence see the appendix of $[\mathbf{J e 3}]$.

The poset $P$ we construct is of size $2^{\aleph_{0}}=\aleph_{2}$. It is $\sigma$-closed and $\aleph_{1}$-Baire, so it is not equivalent to $C_{\omega_{1}}$. But $P \times P$ is nowhere $\aleph_{1}$-Baire; hence by the above corollary $P \times P \simeq C_{\omega_{1}}$. 
Let $A_{\alpha} \subseteq \alpha$ for $\alpha<\omega_{2}$ and $\operatorname{cof}(\alpha)=\omega_{1}$. Consider the following poset $P: p \in P$ iff $p$ is a closed bounded subset of $\omega_{2}$, and $\forall \alpha \in p$ if $\operatorname{cof}(\alpha)=\omega_{1}$, then $p \cap \alpha=A_{\alpha}$. The order is end extension. $P$ will have the desired properties provided we construct $A_{\alpha}$ 's judiciously.

First note that $P$ is obviously $\sigma$-closed. Let us show that $P \times P$ is nowhere $\aleph_{1}$-Baire. To see that consider

$$
D_{\xi}=\{\langle p, q\rangle \in \mathcal{P} \times \mathcal{P} \mid \exists \gamma \in p-q(\operatorname{ot}(p \cap q-\gamma) \geq \xi)\} .
$$

Clearly, $D_{\xi}$ is dense in $\mathcal{P} \times \mathcal{P}$ for $\xi<\omega_{1}$ and $\bigcap_{\xi<\omega_{1}} D_{\xi}=\varnothing$. We now show how to choose $A_{\alpha}$ 's to make $P \aleph_{1}$-Baire.

Let $\left\langle C_{\alpha}\right| \alpha<\omega_{2}$ and $\left.\lim (\alpha)\right\rangle$ be a $\square_{\omega_{1}}$ sequence; i.e., it satisfies

(i) $C_{\alpha}$ is a closed bounded subset of $\alpha$;

(ii) if $\operatorname{cof}(\alpha)<\omega_{1}$, then $\left|C_{\alpha}\right|<\aleph_{1}$;

(iii) if $\gamma$ is a limit point of $C_{\alpha}$, then $C_{\gamma}=C_{\alpha} \cap \gamma$.

Let $\left\langle S_{\alpha} \mid \alpha<\omega_{2} \& \operatorname{cof}(\alpha)=\omega_{1}\right\rangle$ be a $\diamond$ sequence. Let $\left\{X_{\xi} \mid \xi<\omega_{2}\right\}=W$ be an enumeration of closed unbounded subsets of $\omega_{2}$ (remember $\vartheta_{\omega_{2}} \Rightarrow 2^{\aleph_{1}}=\aleph_{2}$ ) such that $X_{\xi} \subseteq \xi$. This induces a well-order on $W$ which we denote by $\prec$. Set $W_{\alpha}=\left\{X_{\xi} \mid \xi<\alpha\right\}$. For $D=\left\langle D_{\xi} \mid \xi<\omega_{1}\right\rangle$, a sequence of subsets of $W, q \in W$, and a limit ordinal $\alpha$, define by induction a decreasing sequence of conditions $q=q_{0} \geq$ $q_{1} \geq \cdots \geq q_{\xi} \geq \cdots$ of length $\gamma_{\alpha}=\operatorname{ot}\left(C_{\alpha}\right)$ in the following way. Let $\left\{\alpha_{\xi} \mid \xi<\gamma_{\alpha}\right\}$ be the increasing enumeration of $C_{\alpha}$. Let $q_{\eta+1}$ be the $\prec$-least $q$ such that $q \in D_{\eta}$, $q \leq q_{\eta}$ and $\sup q \geq \alpha_{\eta}$. If $\eta$ is a limit ordinal, set $q_{\eta}=\operatorname{cl}\left(\bigcup_{\rho<\eta} q_{\rho}\right)$. If $q_{\xi}$ is defined for every $\xi<\gamma_{\alpha}$, let $D(q, \alpha)=\bigcup_{\xi<\gamma_{\alpha}} q_{\xi}$; otherwise let $D(q, \alpha)$ be the empty set.

For $\alpha<\omega_{2}$ and $\operatorname{cof}(\alpha)=\omega_{1}, S_{\alpha}$ codes in some canonical but otherwise unspecified way a sequence of $\omega_{1}$ subsets of $W_{\alpha}, D=\left\langle D_{\xi} \mid \xi<\omega_{1}\right\rangle$ and $q \in W_{\alpha}$. Let then $A_{\alpha}$ be equal to $D(q, \alpha)$. We claim that $\mathcal{P}$ is $\aleph_{1}$-Baire. For if $D=\left\langle D_{\xi} \mid \xi<\omega_{1}\right\rangle$ is a sequence of dense open subsets of $P$ and $q \in P$, using the fact that $\left\langle S_{\alpha}\right| \alpha<$ $\left.\omega_{2} \& \operatorname{cof}(\alpha)=\omega_{1}\right\rangle$ is a $\diamond$ sequence, we find $\alpha<\omega_{2}$ of cofinality $\omega_{1}$ such that

(iv) $\forall s \in W_{\alpha} \forall \beta<\alpha\left(\lim (\beta) \Rightarrow D(\beta, s) \in W_{\alpha}\right)$;

(v) $S_{\alpha}$ codes $\left\langle D_{\xi} \cap W_{\alpha} \mid \xi<\omega_{1}\right\rangle$.

Then it follows from our construction that if $t=A_{\alpha} \cup\{\alpha\}$, we have $t \in \bigcap_{\xi<\omega_{1}} D_{\xi}$ and $t \leq q$. So $P$ is $\aleph_{1}$-Baire.

2. The cut $\&$ choose games. Let $B$ be a Boolean algebra and $\kappa$ a cardinal. The cut \& choose game $\mathcal{G}_{\kappa}(B)$ is played as follows. First Empty picks an $a \in B^{+}$ and an antichain $A_{0}$ of size $\leq \kappa$ such that $a=\bigvee A_{0}$. Then Nonempty chooses $a_{0} \in A_{0}$. Then Empty picks an antichain $A_{1}$ of size $\leq \kappa$ such that $a_{0}=\bigvee A_{1}$, and Nonempty chooses some $a_{1} \in A_{1}$. The game proceeds like this ad infinitum. A sequence $A_{0}, a_{0}, A_{1}, a_{1}, \ldots, A_{n}, a_{n}, \ldots$ is produced where each $A_{n}$ is a partition of $a_{n-1}$, of size $\leq \kappa$ and $a_{n} \in A_{n}$. We say that Nonempty wins iff $\bigwedge_{n<\omega} a_{n} \neq 0$. The game $\mathcal{G}_{\infty}(B)$ is played in the same way but with no restriction on the size of $A_{n}$ 's.

Jech proved [Je3, Theorem 2] that Empty has a winning strategy in $\mathcal{G}_{\kappa}(B)$ iff $B$ is not $\left(\aleph_{0}, \kappa\right)$-distributive. Our first result in this section is that $\mathcal{G}_{\infty}$ and the Banach-Mazur game $\mathcal{G}$ are equivalent in terms of winning strategies. The case of strategies for Empty follows from the above results of Jech. Also, a strategy for Nonempty in $\mathcal{G}(B)$ easily gives one for him in $\mathcal{G}_{\infty}(B)$. So, we are done if we show the following. 
THEOREM 2.1. If Nonempty wins $\mathcal{G}_{\infty}(B)$, then he wins $\mathcal{G}(B)$.

Proof. Let $\sigma$ be a winning strategy for Nonempty in $\mathcal{G}_{\infty}(B)$. We can think of $\sigma$ as a function which, given a partial play $a_{0}, A_{0}, a_{1}, A_{1}, \ldots, A_{n}$, chooses an element $\sigma\left(a_{0}, A_{0}, a_{1}, A_{1}, \ldots, A_{n}\right)$ of $A_{n}$. We describe a strategy for Nonempty in $\mathcal{G}(B)$.

Assume Empty first plays nonzero $a_{0} \in B^{+}$. Consider the set $\Sigma_{a_{0}}=\left\{\sigma\left(a_{0}, A\right) \mid A\right.$ is an antichain $\left.\& \bigvee A=a_{0}\right\}$. We claim that there is nonzero $b_{0} \leq a_{0}$ such that $\Sigma_{a_{0}}$ contains all nonzero elements below $b_{0}$. Otherwise there is an antichain $A$ disjoint form $\Sigma_{a_{0}}$ and $\bigvee A=a_{0}$. But then we have $\sigma(A) \in A \cap \Sigma_{a_{0}}$, a contradiction. We let Nonempty play such a $b_{0} \leq a_{0}$. Empty then plays $a_{1} \leq b_{0}$. Nonempty first chooses an antichain $A_{0}$ such that $\bigvee A_{0}=a_{0}$ and $\sigma\left(a_{0}, A_{0}\right)=a_{1}$. Then he considers the set $\Sigma_{a_{0}, A_{0}}=\left\{\sigma\left(a_{0}, A_{0}, a_{1}, A\right) \mid A\right.$ is an antichain $\left.\& \bigvee A=a_{1}\right\}$. Again, there is a nonzero element $b_{1} \leq a_{1}$ such that $B^{+} \uparrow b_{1} \subseteq \Sigma_{a_{0}, A_{0}}$. We let Nonempty play such a $b_{1}$. Then, again Empty plays $a_{2} \leq b_{1}$. Nonempty first chooses an antichain $A_{1}$ such that $\sigma\left(a_{0}, A_{0}, a_{1}, A_{1}\right)=a_{2}$; then he looks at

$$
\Sigma_{a_{0}, A_{0}, a_{1}, A_{1}, a_{2}}=\left\{\sigma\left(a_{0}, A_{0}, a_{1}, A_{1}, a_{2}, A\right) \mid A \text { antichain } \& \bigvee A=a_{2}\right\}
$$

and defines $b_{2}$ as above. In such a way Nonempty is able to proceed and, given a play $a_{0} \geq b_{0} \geq a_{1} \geq b_{1} \geq \cdots \geq a_{n} \geq \cdots$ in $\mathcal{G}(B)$, simultaneously produce a play $A_{0}, a_{1}, A_{1}, a_{2}, \ldots, A_{n}, a_{n}, \ldots$ in $\mathcal{G}_{\infty}(B)$ such that for every $n \in \omega a_{n}=$ $\sigma\left(a_{0}, A_{0}, a_{1}, A_{1}, \ldots, A_{n}\right)$. But $\sigma$ is a winning strategy for Nonempty in $\mathcal{G}_{\infty}(B)$, hence $\bigwedge_{n \in \omega} a_{n} \neq 0$. We have thus produced a winning strategy for Nonempty in $\mathcal{G}(B)$.

Before we proceed any further let us give an application of what we have just proved. Recall that a $\kappa$-Suslin tree is a tree of size $\kappa$ with no antichainsor chains of size $\kappa$. For regular $\kappa, \kappa$-Suslin trees may or may not exist; see [To]. A $\kappa$-Suslin tree $T$ is called essentially $\sigma$-closed if there is a cub $C \subset \kappa$ such that $T \uparrow C$ is $\sigma$-closed. In the following discussion we assume the reader is familiar with the definition of weakly compact cardinals and some of their basic properties.

THEOREM 2.2. If "ZFC $+\exists$ a weakly compact cardinal" is consistent, then so $i s$ "ZFC + GCH $+\forall \aleph_{2}$-Suslin tree is essentially $\sigma$-closed".

PROOF. Recall the following well-known version of the Levy collapsing partial order $\mathcal{L}_{\kappa}$ of $\kappa$ to $\aleph_{2}$. Elements of $\mathcal{L}_{\kappa}$ are countable functions $p$ satisfying $\operatorname{dom}(p) \subseteq$ $\omega_{1} \times \kappa, \operatorname{ran}(p) \subseteq \kappa$ and $p(\alpha, \beta) \in \beta$ for every $(\alpha, \beta) \in \operatorname{dom}(p)$. The order is reverse inclusion. We show that if GCH holds and $\kappa$ is weakly compact, then for any $\mathcal{L}_{\kappa}$-generic filter $G_{\kappa}, V\left[G_{\kappa}\right]$ is the desired model. We make the following claim in $V\left[G_{\kappa}\right]$, fixing a $\kappa$-Suslin tree $T$.

Claim. There is a $\sigma$-closed poset $P$ such that for every $t \in T$ there is a $P$-name $\dot{b}$ such that $\Vdash_{p}$ " $\dot{b}$ is a branch through $T$ of length $\kappa$ and $t \in \dot{b}$."

PROOF OF CLAIM. Let us work for a moment in the ground model. Fix a name $\dot{T}$ for $T$ such that $\dot{T} \subseteq V_{\kappa}$. Let $M$ be a transitive model of size $\kappa$ of a sufficient fragment of ZFC, which is $<\kappa$ closed and such that $\kappa+1 \cup\left\{\dot{T}, \mathcal{L}_{\kappa}\right\} \subseteq M$. As in Lemma 32.4 of [Je2], there is a transitive model $N$ closed under sequences of lenght $<\kappa$ and elementary embedding $j: M \rightarrow N$ such that $\kappa$ is the least ordinal moved under $j$. By a well-known fact $j$ can be extended to an embedding $j^{*}: M\left[G_{\kappa}\right] \rightarrow N\left[G_{j(\kappa)}\right]$ where $G_{j(\kappa)}$ is $\mathcal{L}_{j(\kappa)}$ generic over $N$ such that $G_{j(\kappa)} \cap \mathcal{L}_{\kappa}=G_{(\kappa)} . G_{j(\kappa)}$ can be 
obtained by forcing with $\mathcal{L}_{j(\kappa)-\kappa}$ over $V\left[G_{\kappa}\right]$, and this poset is $\sigma$-closed; i.e., if $G_{j(\kappa)-\kappa}$ is $\mathcal{L}_{j(\kappa)-\kappa}$ generic over $V\left[G_{\kappa}\right]$, then we can let $G_{j(\kappa)}=G_{\kappa} \times G_{j(\kappa)-\kappa}$. By elementarity, it follows that in $N\left[G_{j(\kappa)}\right]$ every element of $j^{*}(T)$ has arbitrary high extensions. Hence, for every $t \in T$ there is $t^{*} \in j^{*}(T)$ above $j(t)(=t)$ which is on the $\kappa$ th level. But then $b=\left\{s \in T \mid s<_{j^{*}(T)} t^{*}\right\}$ is a $\kappa$-branch through $T$ and $t \in b$. So, $P=\mathcal{L}_{j(\kappa)-\kappa}$ is the required poset. The claim is proved.

Back to the proof of the theorem. Let us now describe a winning strategy for Nonempty in $\mathcal{G}_{\infty}(T)$. Let Empty's first move be $t \in T$ and a maximal antichain below $t, A_{0}$. Since $T$ is a Suslin tree, we may assume, by refining if necessary, that $A_{0}$ consists of elements of $T$ on a fixed level $T_{\alpha_{0}}$ for some $\alpha_{0}<\kappa$.

Nonempty fixes $b$ as in the claim and finds $p_{0} \in P$ such that, for some $t_{0} \in$ $T_{\alpha_{0}}, p_{0} \Vdash_{p}$ " $\dot{b} \cap T_{\alpha_{0}}=\left\{t_{0}\right\}$ ". Empty now plays another level $T_{\alpha_{1}}$ for some $\alpha_{1}>\alpha_{0}$. Nonempty then finds some $p_{1} \leq p_{0}$ and $t_{1} \in T_{\alpha_{1}}$ such that $p_{1} \Vdash " \dot{b} \cap T_{\alpha_{1}}=\left\{t_{1}\right\}$ ". In this way we have a play $t, T_{\alpha_{0}}, t_{0}, T_{\alpha_{1}}, t_{1}, \ldots, T_{\alpha_{n}}, t_{n}, \ldots$. Nonempty along the way constructs a decreasing sequence of elements of $P, p_{0} \geq p_{1} \geq \cdots \geq p_{n} \geq \cdots$, such that $p_{n} \Vdash_{P}$ " $\dot{b} \cap T_{\alpha_{n}}=\left\{t_{n}\right\}$ ". Now, since $\mathcal{P}$ is $\sigma$-closed, there is $p_{\omega} \in \mathcal{P}$ such that $\forall n p_{\omega} \leq p_{n}$. Since $p_{\omega} \Vdash_{p}$ " $\dot{b}$ is a branch of length $\kappa$ ", there must exist $t_{\omega} \in T_{\alpha_{\omega}}$, where $\alpha_{\omega}=\sup _{n \in \omega} \alpha_{n}$ and $p \leq p_{\omega}$, such that $p \Vdash_{\rho}$ " $\dot{b} \cap T_{\alpha_{\omega}}=\left\{t_{\omega}\right\} "$. It is clear now that $\forall n t_{n} \leq t_{\omega}$, so Nonempty has won this game; i.e., we have described a winning strategy for him.

By Theorem 1.1 $T$ has a $\sigma$-closed dense set $D$. We may assume that $D$ is closed in the tree topology; i.e., if $D$ is unbounded below some $t \in T$, then $t \in D$. Let us now force with $T$. If $G_{T}$ is a generic branch through $T$, it is clear that $C_{G_{T}}=\left\{\alpha<\kappa \mid G_{T} \cap T_{\alpha} \subseteq D\right\}$ is closed and unbounded in $\kappa$. Since $T$ is a $\kappa$-cc poset, there exists a cub $C$ in the ground model such that $\Vdash_{T}$ " $C \subseteq \dot{C}_{G_{T}}$ ". Then $T \uparrow C$ is $\sigma$-closed. This finishes the proof.

Let us remark at this point that under the assumption of GCH $+\square_{\omega_{1}}$ Gregory $[\mathbf{G r}]$ has constructed an $\aleph_{2}$-Suslin tree which is essentially non- $\sigma$-closed. This shows that some large cardinal assumption in Theorem 2.2 is necessary. Of course, the consistency of "GCH+ no $\aleph_{2}$-Suslin trees" is a well-known open problem. For some information concerning this problem see [LS and SS].

We now consider the following question: when does the existence of a winning strategy for one player in $\mathcal{G}_{\lambda}(B)$ imply the existence of a winning strategy for the same player in $\mathcal{G}_{\kappa}(B)$ ? In the case of the Empty player this reduces to some well-known problems of distributivity of Boolean algebras, so we concentrate on strategies for Nonempty. The case $\kappa \leq \lambda$ being trivial, we may assume $\lambda<\kappa$. For definiteness we restrict ourselves to the case $\lambda=2$, although most of our results can be generalized to bigger $\lambda$ 's under appropriate assumptions. We first recall some well-known games of Mycielski and Ulam, played on sets rather than Boolean algebras; see for example [KM, p. 249].

The Mycielski game played on a cardinal $\kappa$ runs as follows. Empty splits $\kappa$ into two parts. Nonempty chooses one of the parts, and Empty then splits the chosen part. Nonempty chooses again, and so on. Nonempty wins iff the intersection of the chosen pieces has at least two points.

The Ulam game is similar, except that both players split and choose. Nonempty wins if the intersection of the chosen parts is not empty. 
We need some known facts about these games; see [KM, p. 249, and GJM] for further information and references.

(1) If $\kappa$ is less than the first measurable cardinal, then if Nonempty wins the Mycielski game on $\kappa$, he also wins the Ulam game on $\kappa$.

(2) If $\kappa \leq 2^{\aleph_{0}}$, then Nonempty does not have a winning strategy in the Ulam (and hence Mycielski) game on $\kappa$.

(3) If "ZFC + there is a measurable cardinal" is consistent, then so is "ZFC + Nonempty has a winning strategy in both games played on $\omega_{2} . "$

(4) If for some $\kappa$ Nonempty has a winning strategy in either game on $\kappa$, then there is an inner model with a measurable cardinal.

THEOREM 2.3. Let $B$ be a complete Boolean algebra such that Nonempty has a winning strategy in $\mathcal{G}_{2}(B)$. If there is no winning strategy for Nonempty in the Ulam game on $\kappa$ and $\kappa$ is less than the first measurable cardinal, then Nonempty wins $\mathcal{G}_{\kappa}(B)$.

PROOF. Let us fix a winning strategy $\sigma$ for Nonempty in $\mathcal{G}_{2}(B)$. We can think of $\sigma$ as a function such that, given $a \in B^{+}$and a sequence of partitions of $a$ into two pieces, $\left\langle\left\langle a_{i}^{0}, a_{i}^{1}\right\rangle \mid i \leq n\right\rangle$ it chooses one piece of the last partition-i.e., $\sigma\left(a,\left\langle\left\langle a_{i}^{0}, a_{i}^{1}\right\rangle \mid i \leq n\right\rangle\right)=\varepsilon$, for some $\varepsilon \in\{0,1\}$-in such a way that for every $a$ and infinite sequence of partitions of $a s=\left\langle\left\langle a_{i}^{0}, a_{i}^{1}\right\rangle \mid i<\omega\right\rangle$, setting $\sigma(a, s \mid n+1)=\varepsilon_{n}$ for $n<\omega$, we have that $\bigwedge_{n<\omega} a_{n}^{\varepsilon_{n}} \neq 0$. By a partial play in $\mathcal{G}_{2}(B)$ we mean an element $a$ of $B^{+}$and a finite sequence of partitions as above. We now describe a strategy for Nonempty in $\mathcal{G}_{\kappa}(B)$.

Let Empty play $a \in B^{+}$and an antichain $A_{0}$ of size $\leq \kappa$ such that $\bigvee A_{0}=a$. For $X \subseteq A_{0}$, put $a_{X}^{0}=\bigvee X$ and $a_{X}^{1}=a-a_{X}^{0}$. We claim that there is a partial play $s_{0}$ in $\mathcal{G}_{2}(B)$ which begins with $a$, and a function $f_{0}: P\left(A_{0}\right) \rightarrow 2$ such that whenever $t$ is a play extending $s_{0}$ and $X \subseteq A_{0}$ then $\sigma\left(t^{\frown}\left\langle a_{X}^{0}, a_{X}^{1}\right\rangle\right)=f_{0}(X)$. This means that for plays extending $s_{0}$ and for every $X \subseteq A \sigma$ always chooses the same element of the partition $\left\langle a_{X}^{0}, a_{X}^{1}\right\rangle$.

Assume otherwise. Then we can get a winning strategy for Nonempty in the Ulam game on $A_{0}$ as follows. Along with playing the Ulam game on $A_{0}$, Nonempty simulates a play in $\mathcal{G}_{2}(B)$ in the following way. Suppose at some stage we have $X \subseteq A_{0}$ in the Ulam game and $s$ in the simulated game such that $a_{X}^{0}$ was chosen in some move of $s$; i.e., for some $n<\operatorname{length}(s), s(n)=\left\langle a_{X}^{0}, a_{X}^{1}\right\rangle$ and $\sigma(s \mid n+1)=0$. Now it is Nonempty's turn to split $X$. He first chooses two partial plays $s^{\prime}$ and $s^{\prime \prime}$ extending $s$ such that the last terms of $s^{\prime}$ and $s^{\prime \prime}$ are the same, equal to $\left\langle a_{Y}^{0}, a_{Y}^{1}\right\rangle$ for some $Y \subseteq A_{0}$, and $\sigma\left(s^{\prime}\right)=0$ and $\sigma\left(s^{\prime \prime}\right)=1$. Such $s^{\prime}$ and $s^{\prime \prime}$ exist, lest the claim is true for $s_{0}=s$. Nonempty now splits $X$ into $X \cap Y$ and $X-Y$. If Empty chooses $X \cap Y$, Nonempty extends his simulated play to $s^{\prime}$; if Empty chooses $X-Y$, Nonempty extends his simulated play to $s^{\prime \prime}$. When it is Nonempty's turn to choose between, say $X$ and $A_{0}-X$, he looks at the current simulated play $s$ and chooses $X$ if $\sigma\left(s^{-}\left\langle a_{X}^{0}, a_{X}^{1}\right\rangle\right)=0$, otherwise he chooses $A_{0}-X$. It can easily be checked that in this way we have described a winning strategy for Nonempty in the Ulam game. The claim is proved.

Now, Nonempty fixes $s_{0}$ and $f_{0}$ as claimed. It is clear that $U_{0}=\{X \subseteq$ $\left.A_{0} \mid f_{0}(X)=0\right\}$ is a countably complete ultrafilter on $A_{0}$, and since $\left|A_{0}\right|$ is less than the first measurable cardinal, there must exist a sequence $X_{0}^{0}, X_{1}^{0}, X_{2}^{0}, \ldots$ of 
elements of $\mathcal{U}_{0}$ such that $\bigcap_{n<\omega} X_{n}^{0}$ is a singleton, say $\left\{a_{0}\right\}$. Nonempty fixes such a sequence $\left\{X_{n}^{0}: n<\omega\right\}$ and $a_{0}$. Back in the original game he plays $a_{0}$.

Empty now plays another antichain $A_{1}$ of size $\leq \kappa$ such that $\bigvee A_{1}=a_{0}$ Nonempty looks back at $s_{0}$, extends it first to $s_{0}^{\prime}=\widehat{s_{0}}\left\langle a_{X_{0}^{0}}^{0}, a_{X_{0}^{0}}^{1}\right\rangle$ and, in the same way as above, finds $s_{1}$ extending $s_{0}^{\prime}$ and $f_{1}: \mathcal{P}\left(A_{1}\right) \rightarrow 2$ such that for every play $t$ extending $s_{1}$ and $X \subseteq A_{1}$ we have $\sigma\left(t^{\sim}\left\langle a_{X}^{0}, a_{X}^{1}\right\rangle\right)=f_{1}(X)$, where again for $X \subseteq A_{1} a_{X}^{0}=\bigvee X$ and $a_{X}^{1}=a-a_{X}^{0}$. As above, Nonempty fixes a sequence $X_{0}^{1}, X_{1}^{1}, X_{2}^{1}, \ldots$ of elements of $\mathcal{U}_{1}=\left\{X \subseteq A_{1} \mid f_{1}(X)=0\right\}$ such that $\bigcap_{n<\omega} X_{n}^{1}=\left\{a_{1}\right\}$ and plays as his choice $a_{1} \in \overline{A_{1}}$. Aside, he extends $s_{1}$ to $s_{1}^{\prime}=s_{1}\left\langle a_{X_{1}^{0}}^{0}, a_{X_{1}^{0}}^{1}\right\rangle-\left\langle a_{X_{0}^{1}}^{0}, a_{X_{0}^{1}}^{0}\right\rangle$. It is clear now that Nonempty will be able to proceed in this fashion and if $A_{0}, a_{0}, A_{1}, a_{1}, \ldots, A_{n}, a_{n}, \ldots$ is the play produced, he would have a corresponding play in $\mathcal{G}_{2}(B), s=\bigcup_{n<\omega} s_{n}=\left\langle a,\left\langle b_{i}^{0}, b_{i}^{1}\right\rangle \mid i<\omega\right\rangle$ such that if $\sigma\left(a,\left\langle b_{i}^{0}, b_{i}^{1}\right\rangle \mid i \leq n\right)=\varepsilon_{n}$ we have $\bigwedge_{n<\omega} b_{n}^{\varepsilon_{n}} \leq \bigwedge_{n<\omega} a_{n}$. But, $\sigma$ is a winning strategy for Nonempty in $\mathcal{G}_{2}(B)$, so we must have $\bigwedge_{n<\omega} b_{n}^{\varepsilon_{n}} \neq 0$, and hence $\bigwedge_{n<\omega} a_{n} \neq 0$. We have thus described a winning strategy for Nonempty in $g_{\kappa}(B)$.

COROllary 2.4. Let $B$ be a $c B a$ of density $\leq 2^{\aleph_{0}}$. Assume Nonempty wins $\mathcal{G}_{2}(B)$. Then $B$ has a $\sigma$-closed dense set.

PROOF. Follows directly from Theorem 2.3, Theorem 2.1 and Corollary 1.3.

COROLlaRY 2.5. There is an $\left(\aleph_{0}, 2\right)$-distributive complete Boolean algebra $B$ such that Nonempty does not have a winning strategy in $\mathcal{G}_{2}(B)$.

PROOF. By the above results it is enough to give an example of a partially ordered set of size $2^{\aleph_{0}}$ which is $\sigma$-Baire but does not have a $\sigma$-closed dense set. A standard example of this is the poset for shooting a cub set through a stationary costationary subset of $\omega_{1}$ (see [Je3, Example 3]). We shall instead give an example of a poset $P$ which is also proper and hence does not destroy stationary subsets of $\omega_{1}$ (see [Sh]). This example may have some interest of its own.

Fix for each limit ordinal $\alpha<\omega_{1}$, a cofinal subset $A_{\alpha}$ of $\alpha$ of order type $\omega$. Let $p \in P$ iff $p$ is a closed bounded subset of $\omega_{1}$ and $\forall \alpha \in \lim \left(\omega_{1}\right)\left[A_{\alpha} \cap p\right.$ is finite $]$. Say that $p \leq q$ iff $p$ is an end extension of $q$; i.e., $p \cap(\max (q)+1)=q$.

Claim. $P$ is $\sigma$-Baire and proper.

PROOF. Let $\theta$ be a large-enough regular cardinal. $H_{\theta}$ is the set of sets hereditarily of size $<\theta$. By a standard argument it is enough to prove the following.

Subclaim. Suppose $M$ is a countable elementary submodel of $\left(H_{\theta}, \varepsilon\right)$ such that $P \in M$, and $p \in P \cap M$. If $D \in M$ is a dense subset of $P$, then there exists $q \in D \cap M$ such that $q \leq p$ and $q \cap A_{\delta}=p \cap A_{\delta}$, where $\delta=M \cap \omega_{1}$.

ProOF. Let $M, \delta, p$ and $P$ be as in the subclaim. We can define by induction a sequence $\left\langle q_{\alpha} \mid \alpha<\omega_{1}\right\rangle$ of elements of $P$ such that

(1) $\forall \alpha<\omega_{1}\left\{q_{\alpha} \leq p\right.$ and $\left.q_{\alpha} \in D\right]$,

(2) $\alpha<\beta \rightarrow \max \left(q_{\alpha}-p\right)<\min \left(q_{\beta}-p\right)$.

By elementarity there is such a sequence in $M$. Since $A_{\delta}$ has order type $\omega$, there exists $\xi<\delta$ such that $\left(q_{\xi}-p\right) \cap A_{\delta}=\varnothing$, but then $q=q_{\xi} \in M$ is as required. The subclaim is proved.

Let us now assume $D$ is a dense, $\sigma$-closed subset of $P$. Let $M \prec\left(H_{\theta}, \varepsilon\right)$ be countable such that $\mathcal{P}, D \in M$. Let $\delta=M \cap \omega_{1}$. We build by induction a decreasing 
sequence of elements of $D \cap M, q_{0} \geq q_{1} \geq \cdots \geq q_{n} \geq \cdots n<\omega$. Suppose $q_{n}$ is defined. Since $\max \left(q_{n}\right)<\delta$, there is $\delta_{n} \in A_{\delta}$ such that $\max \left(q_{n}\right)<\delta_{n}$. Let $q_{n+1} \in D \cap M$ extend $q_{n} \cup\left\{\delta_{n}\right\}$. It is now clear that $\left(\bigcup_{n<\omega} q_{n}\right) \cap A_{\delta}$ is infinite. So, the sequence $\left\langle q_{n} \mid n<\omega\right\rangle$ cannot have a lower bound in $\mathcal{P}$. This contradiction finishes the proof.

Proposition 2.6. Let $B$ be a complete nonatomic Boolean algebra. Assume Nonempty wins $\mathcal{G}_{2}(B)$. Then $B$ has an antichain of size $2^{\aleph_{0}}$.

ProOF. If $B$ satisfies the $2^{\aleph_{0}}$-chain condition, then Nonempty wins $\mathcal{G}_{\infty}(B)$, and hence $\mathcal{G}(B)$. Then as in the proof of Theorem 1.1, we can construct a Cantor tree of partial plays in $\mathcal{G}(B)$ such that each branch has nonzero infimum and elements corresponding to different branches are disjoint. There are $2^{\aleph_{0}}$ branches through this tree, so we can get an antichain of $2^{\aleph_{0}}$, thus contradicting the assumption that $B$ satisfies the $2^{\aleph_{0}}$-chain condition.

As Jeck remarks in [Je3], Prikry forcing is an example of an algebra which is not $\left(\aleph_{0}, \infty\right)$-distributive, but Nonempty wins $\mathcal{G}_{2}$. Another candidate is Namba forcing (see [Na or Je2, p. 289]). Here we have the following result of Shelah [Sh, Chapter $\mathrm{XII}]$.

THEOREM 2.7. The following are equivalent.

(i) Nonempty wins the Mycielski game on $\omega_{2}$,

(ii) Nonempty wins $\mathcal{G}_{2}(B)$ for some complete Boolean algebra $B$ which is not $\left(\aleph_{0}, \aleph_{2}\right)$-distributive,

(iii) Nonempty wins $\mathcal{G}_{2}$ on the Namba algebra.

By generalizing the proof of this theorem with arbitrary $\kappa$ instead of $\omega_{2}$ and using Theorem 2.3 and fact (4) above, we can conclude that if there is no inner model with a measurable cardinal, the games $\mathcal{G}_{2}$ and $\mathcal{G}$ are equivalent in terms of the existence of winning strategies for Nonempty.

3. What if both players cut and choose? We now modify the cut \& choose game $\mathcal{G}_{2}$ by requiring Nonempty to cut as well. Call this new game $\mathcal{G}_{2}^{*}$. It is the Boolean algebraic version of the Ulam game. Concerning the existence of winning strategies for Empty, we have the following.

THEOREM 3.1. Let $B$ be an atomless complete Boolean algebra. Then Empty has a winning strategy in $\mathcal{G}_{2}^{*}(B)$ iff $B$ is not $\left(\aleph_{0}, 2\right)$-distributive.

Note that if $B$ has an atom, then Empty wins by playing it. So it is natural to restrict ourselves to nonatomic algebras.

PROOF. If $B$ is not $\left(\aleph_{0}, 2\right)$-distributive, then Empty wins easily; in fact, he even wins $\mathcal{G}_{2}(B)$. So let us assume that $B$ is $\left(\aleph_{0}, 2\right)$-distributive and there is a winning strategy $\sigma$ for Emtpy. We may without loss of generality assume that $\sigma(\langle\rangle)$ is a partition of 1 ; for if $\sigma(\langle\rangle)=\left\langle a_{0}, a_{1}\right\rangle$ we simply restrict ourselves to $B \uparrow\left(a_{0} \vee a_{1}\right)$.

LEMMA 3.2. B has an uncountable antichain.

PROOF. Let $\theta$ be a regular, large enough cardinal and $H_{\theta}$ the set of all sets of hereditary size less than $\theta$. We fix a countable $M \prec\left(H_{\theta}, \varepsilon\right)$ such that $\sigma, B \in M$. Let $\left\langle\left\langle a_{n}^{0}, a_{n}^{1}\right\rangle \mid n<\omega\right\rangle$ enumerate all partitions of 1 into two pieces which are in $M$. Since we assume $B$ is $\left(\aleph_{0}, 2\right)$-distributive, there is some $f \in 2^{\omega}$ such that 
$a=\bigwedge_{n<\omega} a_{n}^{f(n)} \neq 0$. We now try to build a run of the game in which Empty follows $\sigma$, which is contained in $M$ and such that at each stage the chosen piece contains $a$.

More formally, let $\sigma(\langle\rangle)=\left\langle b_{0}^{0}, b_{0}^{1}\right\rangle$. Since $\sigma \in M, b_{0}^{0}$ and $b_{0}^{1}$ are in $M$, so there is $\varepsilon_{0} \in\{0,1\}$ such that $a \leq b_{0}^{\varepsilon_{0}}$. If possible, Nonempty finds $b_{1}^{0}, b_{1}^{1} \in M$ which partition $b_{0}^{\varepsilon_{0}}$ and such that if $\sigma$ chooses and splits $b_{1}^{\varepsilon_{1}}$, then $a \leq b_{1}^{\varepsilon_{1}}$; i.e. Nonempty tries to make sure that at each stage, the chosen piece contains $a$. So, if $\sigma$ tells Empty to choose $b_{1}^{\varepsilon_{1}}$ and split it into $b_{2}^{0}$ and $b_{2}^{1}$, then Nonempty will choose $b_{2}^{\varepsilon_{2}}$ for $\varepsilon_{2} \in\{0,1\}$ in such a way that $a \leq b_{2}^{\varepsilon_{0}}$. Again, if possible he will split it into $b_{3}^{0}$ and $b_{3}^{1}$ such that if Empty chooses and splits $b_{3}^{\varepsilon_{3}}$ for some $\varepsilon_{3} \in\{0,1\}$ we then have $a \leq b_{3}^{\varepsilon_{3}}$. It is clear that Nonempty cannot play like this ad infinitum, for we would then have $a \leq \bigwedge_{n<\omega} b_{n}^{\varepsilon_{n}}$, contradicting the fact that $\sigma$ is a winning strategy for Empty. So, it must happen that at some stage $n$ we arrive at a partial play according to $\sigma, s=\left\langle\left\langle b_{0}^{0}, b_{0}^{1}\right\rangle, \ldots,\left\langle b_{2 n}^{0}, b_{2 n}^{1}\right\rangle\right\rangle$ such that for some $\varepsilon_{2 n} \in\{0,1\}$ we have $a \leq b_{2 n}^{\varepsilon_{2 n}}$ and for every partition $\left\langle b^{0}, b^{1}\right\rangle$ of $b_{2 n}^{\varepsilon_{2 n}}$ which is in $M, \sigma\left(s^{-}\left\langle b^{0}, b^{1}\right\rangle\right)$ chooses and splits $b^{i}(i \in\{0,1\})$ such that $a \leq b^{1-i}$.

Now, let $I=\left\{c \leq b \mid \sigma\left(s^{-}\langle c, b-c\rangle\right)\right.$ is a partition of $\left.c\right\}$. We claim that $I$ is a countably complete maximal ideal in $B \uparrow b$. Note that for $c \in B \cap M, c \in I$ iff $c \wedge a=0$. This implies at the same time that $I$ is an ideal and that it is maximal. If $I$ were not countably complete, by elementarity of $M$ there would be a subset $X=\left\{b_{n} \mid n \in \omega\right\}$ of $I$ such that $\bigvee_{n \in \omega} b_{n} \notin I$ and $X \in M$. Then for every $n<\omega$ we have $b_{n} \in M \cap I$, and hence $b_{n} \wedge a=0$. This implies $\left(\bigvee_{n<\omega} b_{n}\right) \wedge a=0$. So, $\bigvee_{n \in \omega} b_{n} \in I$, a contradiction.

Finally, let $A$ be a maximal antichain in $B \mid b$ consisting of elements of $I$. Since $I$ is countably complete, $A$ cannot be countable. The lemma is proved.

PROOF OF THEOREM 3.1. Let $A$ be a maximal antichain in $B$ of size $\aleph_{1}$. Let us fix $\left\{W_{n} \mid n<\omega\right\}$ a countable, point separating family of subsets of $A$. It can be obtained, for example, as follows: fix $f: A \rightarrow 2^{\omega}$ which is 1-1 and let $W_{n}=\{a \mid f(a)(n)=0\}$. Let us define $w_{n}=\bigvee W_{n}$ for $n<\omega$ and $W=\left\{w_{n} \mid n<\omega\right\}$.

Fix $a \in A$. We define by induction sequences $\tau_{s} ; s \in 2^{<\omega}$ of partial plays according to $\sigma$, and $w_{s} ; s \in 2^{<\omega}$ of elements of $\mathcal{W}$, as long as possible in the following way.

Suppose we have defined $\tau_{s}$ and $w_{s}$ for all $s \in 2^{\leq n}$ for some $n<\omega$, and fix some $s \in 2^{n}$ and $\varepsilon \in\{0,1\}$. Let $\sigma\left(\tau_{s}\right)=\left\langle b_{s-0}, b_{s-1}\right\rangle$. If there exists $w \in \mathcal{W}$ such that $a \leq w$ and $\sigma\left(\tau_{s}\left\langle b_{s-0}, b_{s-1}\right\rangle-\left\langle b_{s \frown \varepsilon} \wedge w, b_{s-\varepsilon}-w\right\rangle\right)$ is a partition of $b_{s\urcorner \varepsilon} \wedge w$, we let

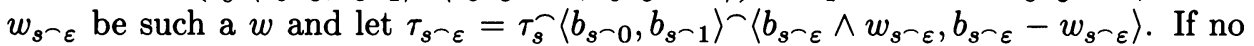
such $w$ exists, we terminate the construction.

If we could define $w_{s}$ and $\tau_{s}$ for every $s \in 2^{<\omega}$, we would get a sequence $\left\langle b_{s}\right| s \in$ $\left.2^{<\omega}\right\rangle$ such that for every $n<\omega, a \leq \bigvee\left\{b_{s} \mid s \in 2^{n}\right\}$. Since $B \mid a$ is $\left(\aleph_{0}, 2\right)$ distributive, there would exist an $f \in 2^{\omega}$ such that $\bigwedge\left\{b_{f \mid n} \mid n<\omega\right\} \neq 0$. Then $\bigcup\left\{\tau_{f \mid n}: n<\omega\right\}$ would be a play in which Empty lost even though he followed his winning strategy $\sigma$, a contradiction.

So, for every $a \in A$, there is for some $s \in 2^{<\omega}$ a partial play $\tau_{s}$ consistent with $\sigma$ such that for some $\varepsilon \in\{0,1\}, w_{s^{\wedge} \varepsilon}$ and $\tau_{s\urcorner \varepsilon}$ cannot be defined. Now, using the fact that $\left\{W_{n} \mid n<\omega\right\}$ is a point separating family of subsets of $A$, we see that $\tau_{s}$ and $\varepsilon$ uniquely determine $a$. Namely, if $\sigma\left(\tau_{s}\right)=\left\langle b_{s-0}, b_{s-1}\right\rangle$, we have

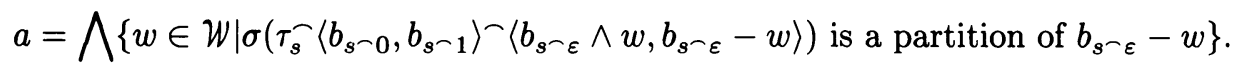


Since $\mathcal{W}$ is countable, there are only countably many partial plays $\tau_{s}$ as above, so we get a 1-1 function from $A$ into a countable set. This contradiction finishes the proof.

How about stategies for Nonempty? If $B$ is such that Nonempty wins $\mathcal{G}_{2}(B)$ and the cardinality of $B$ is less than the first measurable cardinal, then Nonempty wins $\mathcal{G}_{2}^{*}(B)$ as well. This can be proved similarly to Theorem 2.3. Thus if we want an example of an algebra $B$ such that Nonempty wins $\mathcal{G}_{2}(B)$ but not $\mathcal{G}_{2}^{*}(B)$, we have to assume the existence of measurable cardinals.

For $\kappa$ a measurable cardinal and $\mathcal{U}$ a normal $\kappa$-complete ultrafilter on $\kappa$, Prikry forcing is the following partial order $P$; see $[\mathbf{P r}]$. Elements of $P$ are pairs $\langle s, A\rangle$, where $s \in \kappa^{<\omega}, A \in \mathcal{U}$ and $\max \operatorname{ran}(s)<\min A$. We say that $\langle s, A\rangle \leq\langle t, B\rangle$ if $s \supseteq t, A \subseteq B$ and $\operatorname{ran}(s-t) \subseteq B$. Let $B$ be the completion of $P$. We already know that Nonempty wins $\mathcal{G}_{2}(B)$. But does he win $\mathcal{G}_{2}^{*}(B)$ ? It turns out that the answer depends on the model of set theory but not on the particular ultrafilter used.

THEOREM 3.3. Nonempty has a winning strategy in $\mathcal{G}_{2}^{*}(B)$ iff he has one in the Ulam game on $\kappa$.

Proof. We shall prove only the 'if' part. The proof of the 'only if' part is similar but somewhat messier.

Let us recall the following basic fact about Prikry forcing $[\mathbf{P r}]$. If $\langle s, A\rangle \in \mathcal{P}$ and $\left\langle a^{0}, a^{1}\right\rangle$ is a partition of 1 , then there is $A^{\prime} \subseteq A$ and $i \in\{0,1\}$ such that $A^{\prime} \in \mathcal{U}$ and $\left\langle s, A^{\prime}\right\rangle \leq a^{i}$. So, let $\sigma$ be a winning strategy for Nonempty in the Ulam game on $\kappa$. We think of $\sigma$ as a function which, given a finite partial play in the Ulam game, $s=\left\langle\left\langle X_{0}^{1}, X_{0}^{1}\right\rangle, \ldots,\left\langle X_{n}^{0}, X_{n}^{1}\right\rangle\right\rangle$ chooses $\sigma(s)=\left\langle X_{n+1}^{0}, X_{n+1}^{1}\right\rangle$, a partition of $X_{n}^{\varepsilon}$ for some $\varepsilon \in\{0,1\}$.

As usual, along with playing $\mathcal{G}_{2}^{*}(B)$, Nonempty will simulate a play in the Ulam game where he uses his strategy $\sigma$. For $\alpha<\kappa$ let $\bar{p}_{\alpha}=\langle\{\alpha\}, \kappa-(\alpha+1)\rangle \in \mathcal{P}$ and define for $X \subseteq \kappa, p_{X}=\bigvee\left\{\bar{p}_{\alpha} \mid \alpha \in X\right\}$.

Let Empty's first move be $\left\langle a_{0}^{0}, a_{0}^{1}\right\rangle$, a partition of some $a \in B^{+}$. We may without loss of generality assume $a=1$. For each $\alpha<\kappa$, Nonempty finds $A_{0}^{\alpha} \in \mathcal{U}$ and $\varepsilon_{0}(\alpha) \in\{0,1\}$ such that $\left\langle\{\alpha\}, A_{0}^{\alpha}\right\rangle \leq a_{0}^{\varepsilon_{0}(\alpha)}$. Let $X_{0}^{\varepsilon}=\left\{\alpha<\kappa \mid \varepsilon_{0}(\alpha)=\varepsilon\right\}$ for $\varepsilon=0,1$. Empty's simulated move in the Ulam game is $\left\langle X_{0}^{0}, X_{0}^{1}\right\rangle$. Then Nonempty looks at $\sigma\left(\left\langle X_{0}^{0}, X_{0}^{1}\right\rangle\right)=\left\langle X_{1}^{0}, X_{1}^{1}\right\rangle$ a partition of $X_{0}^{\varepsilon_{0}}$ for some $\varepsilon_{0} \in\{0,1\}$. In $\mathcal{G}_{2}^{*}(B)$ Nonempty chooses $a_{0}^{\varepsilon_{0}}$ and splits it into $a_{1}^{0}=a_{0}^{\varepsilon_{0}} \wedge p_{X_{1}^{0}}$ and $a_{1}^{1}=a_{0}^{\varepsilon_{0}}-p_{X_{1}^{0}}$. Note that $a_{0}^{\varepsilon_{0}} \wedge p_{X_{1}^{1}} \leq a_{1}^{1}$. So, assume now Empty chooses $a_{1}^{\varepsilon_{1}}$ and splits it into $a_{2}^{0}$ and $a_{2}^{1}$. Then for every $\alpha \in X_{1}^{\varepsilon_{1}}$ we find $A_{1}^{\alpha} \subseteq A_{0}^{\alpha}$ such that $\left\langle\{\alpha\}, A_{1}^{\alpha}\right\rangle \leq a_{2}^{\varepsilon_{2}(\alpha)}$ for some $\varepsilon_{2}(\alpha) \in\{0,1\}$. Let $\varepsilon=0,1 X_{2}^{\varepsilon}=\left\{\alpha \in X_{1}^{\varepsilon_{1}} \mid \varepsilon_{2}(\alpha)=\varepsilon\right\}$. In the simulated Ulam game Empty's move is to choose $X_{1}^{\varepsilon_{1}}$ and split it into $X_{2}^{0}$ and $X_{2}^{1}$. Then, $\sigma$ says Nonempty has to choose $X_{2}^{\varepsilon_{2}}$ and split it into $X_{3}^{0}$ and $X_{3}^{1}$. Back in $\mathcal{G}_{2}^{*}(B)$ Nonempty chooses $a_{2}^{\varepsilon_{2}}$ and splits it into $a_{3}^{0}=a_{2}^{\varepsilon_{2}} \wedge p_{X_{3}^{0}}$ and $a_{3}^{1}=a_{2}^{\varepsilon_{2}}-p_{X_{3}^{0}}$. Again we have $a_{3}^{1} \leq a_{2}^{\varepsilon_{2}} \wedge p_{X_{3}^{1}}$. The inductive definition of a strategy for Nonempty in $\mathcal{G}_{2}^{*}(B)$ should be clear from this.

In the end the intersection of the chosen $X_{n}^{\varepsilon_{n}}$ 's is nonempty. Let $\alpha \in \bigcap_{n<\omega} X_{n}^{\varepsilon_{n}}$ and let $A^{\alpha}=\bigcap_{n<\omega} A_{n}^{\alpha}$. Then we have $\left\langle\{\alpha\}, A^{\alpha}\right\rangle \leq \bigwedge_{n<\omega} a_{n}^{\varepsilon_{n}}$, and hence Nonempty has won this run of $\mathcal{G}_{2}^{*}(B)$. We have thus described a winning strategy for him. 
The question whether Nonempty wins the Ulam game on a measurable cardinal $\kappa$ has already been considered. We have the following results, due to Solovay, and Magidor and Solovay, respectively.

THEOREM 3.4. (1) If $V=L[\mu]$, where $\mu$ is the (unique) normal measure on $\kappa$, then Nonempty does not have a winning strategy in the Ulam game on $\kappa$.

(2) If "ZFC $+\kappa$ is measureable" is consistent, then so is " $\mathrm{ZFC}+\kappa$ is measurable and Nonempty wins the Ulam game on $\kappa "$.

Since both of these results have not been published, let us say a few words about their proofs. For more information see [GJM; KM, p. 249; Ku and KP].

SKETCH OF PROOF. (1) Assume $V=L[\mu]$ and $\sigma$ is a winning strategy for Nonempty in the Ulam game on $\kappa$. Consider the following forcing notion $\mathcal{Q}$. Elements of $\mathcal{Q}$ are finite partial plays consistent with $\sigma$. The order is end extension. If $G$ is $\mathcal{Q}$ generic over $V$, it defines in a natural way an ultrafilter in $P^{V}(\kappa)$; call it $\mathcal{W}$. It follows from genericity that $\mathcal{W}$ is different from all old ultrafilters on $\kappa$. One can form the generic ultrapower $V^{\kappa} / \mathcal{W}$ as in [So] and, using the fact that $\sigma$ is a winning strategy for Nonempty, show that it is well-founded. So, in $V^{\mathcal{Q}}$ we have an elementary embedding $j: V \rightarrow M$, where $M$ is the transitive class isomorphic to $V^{\kappa} / \mathcal{W}$. Following $[\mathbf{K u}, \S 6]$, one can show that $\kappa$ is the least ordinal moved and so $\mathcal{W}$ is $V$ - $\kappa$-complete. But then by Theorem 7.1 of $[\mathbf{K u}] \mathcal{W}$ is in $V$, contradiction.

(2) Assume that $\kappa$ is measurable and there is a forcing notion $R$ which is $\sigma$-closed and $\kappa$-Baire, and such that for some $\dot{\mathcal{U}} \in V^{R}, \mathbb{H}_{R}$ " $\dot{\mathcal{U}}$ is a new countably complete ultrafilter on $\kappa$ ". This can be proved consistent using the Kunen-Paris method (see $[\mathbf{K P}]$ ).

We describe a strategy for Nonempty. Along with playing the Ulam game on $\kappa$, Nonempty builds a decreasing sequence of elements of $R, p_{0} \geq p_{1} \geq \cdots \geq$ $p_{n} \geq \cdots n<\omega$ such that if $A_{n}$ is the chosen piece at the $n$th stage of the game, then $p_{n} \Vdash$ " $A_{n} \in \dot{U}$ ". If Empty splits $A_{n}$ into $A_{n}^{0}$ and $A_{n}^{1}$, then Nonempty finds $p_{n+1} \leq p_{n}$ such that for some $\varepsilon \in\{0,1\} p_{n+1} \Vdash_{R}$ " $A_{n}^{\varepsilon} \in \dot{\mathcal{U}}$ ", and he then sets $A_{n+1}=A_{n}^{\varepsilon}$. When it is Nonempty's turn to split $A_{n}$, he uses the fact that $p_{n} \Vdash_{R}$ " $\dot{\mathcal{U}}$ is a new ultrafilter" to find a partition $A_{n}^{0}, A_{n}^{1}$ of $A_{n}$ and conditions $p_{n}^{0}, p_{n}^{1} \leq p_{n}$ such that $p_{n}^{\varepsilon} \Vdash_{R}$ " $A_{n}^{\varepsilon} \in \dot{\mathcal{U}}$ " for $\varepsilon \in\{0,1\}$. If Empty now chooses $A_{n+1}=A_{n}^{\varepsilon}$ for some $\varepsilon \in\{0,1\}$, Nonempty sets $p_{n+1}=p_{n}^{\varepsilon}$.

When the game is over, since $R$ is $\sigma$-closed, we can find a condition $p \in R$ such that $\forall n p \leq p_{n}$. Then $p \Vdash_{R}$ " $\bigcap_{n \in \omega} A_{n} \in \dot{\mathcal{U}}$ "; hence $\bigcap_{n \in \omega} A_{n} \neq \varnothing$.

Let us finally remark that, following the idea of the last proof, one can give another example of an algebra $B$ such that Nonempty wins $\mathcal{G}_{2}^{*}(B)$ but Empty wins $\mathcal{G}_{\infty}(B)$. Namely, let $\kappa, R$ and $\dot{U}$ be as in the proof of part (2) of the above theorem, and assume moreover that $\Vdash_{R}$ " $\dot{U}$ is normal". Consider in $V^{R}$ Prikry forcing $P$, using $\dot{U}$ as the ultrafilter. Then look at the iterated forcing $R * \dot{P}$. By an argument similar to the above, we can show that Nonempty wins $\mathcal{G}_{2}^{*}(B)$, where $B$ is the completion of $R * \dot{P}$.

Do large cardinals, for example supercompact, imply the existence of a complete $\mathrm{Ba} B$ such that Nonempty wins $\mathcal{G}_{2}^{*}(B)$, but Empty wins $\mathcal{G}_{\infty}(B)$ ? 


\section{REFERENCES}

[BPS] B. Balcar, J. Pelant and P. Simon, The space of ultrafilters on $N$ covered by nowhere dense sets, Fund. Math. 110 (1980), 11-24.

[De] K. Devlin, Constructibility, Springer-Verlag, Berlin, 1984.

[Fo] M. Foreman, Games played on Boolean algebras, J. Symbolic Logic 48 (1983), 714-723.

[G] C. Gray, Iterated forcing from the strategic point of view, Ph.D. Thesis, Univ. of California, Berkeley, 1980.

[GJM] F. Galvin, T. Jech and M. Magidor, An ideal game, J. Symbolic Logic 43 (1978), 284-292.

[Gr] J. Gregory, Higher Souslin trees and the generalized continuum hypothesis, J. Symbolic Logic 41 (1976), 663-671.

[Je1] T. Jech, The game theoretic property of Boolean algebras, Logic Colloquium 77 (A. Macintyre et al., eds.), North-Holland, Amsterdam, 1978, pp. 135-144.

[Je2] _ Set theory, Academic Press, New York, 1978.

[Je3] _ More game theoretic properties of Boolean algebras, Ann. Pure Appl. Logic 26 (1984), 11-29.

[KM] A Kanamori and M. Magidor, The evolution of large cardinal axioms in set theory, Higher Set Theory (G. H. Miller and D. S. Scott, eds.), Lecture Notes in Math., vol. 669, Springer-Verlag, Berlin, 1978.

$[\mathrm{Ku}] \mathrm{K}$. Kunen, Some applications of iterated ultrapowers in set theory, Ann. Math. Logic 1 (1970), 179-227.

[KP] K. Kunen and J. Paris, Boolean extensions and measurable cardinals, Ann. Math. Logic 2 (1971), 359-378.

[LS] R. Laver and S. Shelah, The $\omega_{2}$-Souslin hypothesis, Trans. Amer. Math. Soc. 264 (1981), 411-417.

[Ma] D. Mauldin, ed., The Scottish book, Birkhäuser, Boston, Mass., 1981.

[My] J. Mycielski, Problem 446, Colloq. Math. 11 (1963), 139.

[Na] K. Namba, Independence proof of $\left(\omega, \omega_{\alpha}\right)$-distributive law in complete Boolean algebras, Comment. Math. Univ. St. Paul. 19 (1970), 1-12.

[Pr] K. Prikry, Changing measurable into accessible cardinals, Dissertationes Math. 68 (1970), $5-52$.

[Sh] S. Shelah, Proper forcing, Lecture Notes in Math., vol. 940, Springer-Verlag, Berlin, 1982.

[SS] S. Shelah and L. Stanley, A "black-box" theorem for morasses, with applications: superSouslin trees and generalized Martin's axiom, Israel J. Math. 43 (1982), 185-224.

[So] R. Solovay, Real-valued measurable cardinals, Axiomatic Set Theory (D. S. Scott, ed.), Proc. Sympos. Pure Math., vol. 13, part 1, Amer. Math. Soc., Providence, R. I., 1971, pp. $397-428$.

[To] S. Todorcević, Trees and linearly ordered sets, Handbook of Set-Theoretic Topology ( $\mathrm{K}$. Kunen and J. Vaughan, eds.), North-Holland, Amsterdam, 1984.

[U1] S. Ulam, SIAM Rev. 6 (1964), 347.

[Vo1] P. Vojtás, A transfinite Boolean game and a generalization of Kripke's embedding theorem, General Topology and its Relations to Modern Analysis and Algebra. V (Proc. Fifth Prague Sympos., 1981), Helderman Verlag, Berlin, 1982, pp. 663-668.

[Vo2] _ Game properties of Boolean algebras, Comment. Math. Univ. Carolin. 24 (1983), 349-369. 53706

Department of Mathematics, University of Wisconsin, Madison, Wisconsin

Current address: Department of Mathematics, California Institute of Technology, Pasadena, California 91125 